\title{
Comparación de las escalas EQ-5D y FACT-G en la evaluación de la calidad de vida en pacientes colombianos con cáncer
}

\author{
Comparision of EQ-5D and FACT-G Scales in the Eualuation of Quality \\ of Life in Colombian Cancer Patients \\ Comparação das escalas EQ-5D e FACT-G na avaliação da qualidade de vida \\ em pacientes colombianos com câncer
}

\author{
Juliet Valdelamar Jiménez, Adriana Valdelamar Jiménez, \\ Luis Fontibón Vanegas, Lorena Acosta Hernández* \\ Instituto Nacional de Cancerología, Colombia \\ Ricardo Sánchez Pedraza* \\ Instituto Nacional de Cancerología y Universidad Nacional de Colombia
}

Doi: dx.doi.org/10.12804/ap133.03.2015.04

\section{Resumen}

Objetivo: Evaluar patrones de correlaciones entre las diferentes dimensiones de una escala que mide la calidad de vida (FACT-G) y otra que mide la salud autopercibida y la calidad de vida (EQ-5D) en pacientes con cáncer. Material y métodos: Estudio descriptivo que evaluó a 114 pacientes con cáncer de mama $(40=$ $35.09 \%)$, gástrico $(26=22.8 \%)$, próstata $(22=19.3 \%)$, recto $(17=14.91 \%)$, a quienes se les aplicó las escalas FACT-G y EQ-5D. Se utilizaron técnicas de estadística descriptiva para resumir variables, métodos de análisis multivariado, análisis factorial y escalamiento multidimensional. Resultados: Las dimensiones que mostraron valores de correlación aceptable fueron bienestar físico y emocional de la FACT-G con angustia y depresión de la escala EQ-5D. El análisis factorial arrojó dos factores: (a) angustia/depresión de la EQ-5D, que se agrupa mejor con los componentes de la FACT-G, y (b) síntomas físicos con los componentes de la EQ-5D. El escalamiento multidimensional sugirió que cada escala midió dimensiones diferentes del constructo calidad de vida. Conclusión: La evaluación de calidad de vida de la FACT-G parece orientarse a aspectos emocionales y de interacción social; mientras que la de EQ-5D se orienta a aspectos de bienestar físico y funcionalidad. Palabras clave: calidad de vida; EQ-5D; FACT-G; cáncer.

\section{Abstract}

Objective: To evaluate patterns of correlations between the different dimensions of a scale measuring quality of life (FACT-G) and one that measures perceived

* Juliet Valdelamar Jiménez, Adriana Valdelamar Jiménez, Luis Fontibón Vanegas, Lorena Acosta Hernández, Instituto Nacional de Cancerología; Ricardo Sánchez Pedraza, Instituto Nacional de Cancerología y Universidad Nacional de Colombia.

La correspondencia relacionada con este artículo debe ser enviada a Juliet Valdelamar Jiménez. Correo electrónico: jvaldelamar@ cancer.gov.co

Cómo citar este artículo: Valdelamar, J., Valdelamar, A., Fontibón, L., Acosta, L. \& Sánchez, R. (2015). Comparación de las escalas EQ-5D y FACT-G en la evaluación de la calidad de vida en pacientes colombianos con cáncer. Avances en Psicología Latinoamericana, 33(3), 413-421. doi: dx.doi.org/10.12804/apl33.03.2015.04 
health and quality of life (EQ-5D) in cancer patients. Methods: A descriptive study that evaluated 114 patients with confirmed cancer of breast ( $40=35.09 \%$ ), gastric $(26=22.8 \%)$, prostate $(22=19.3 \%)$, colon-rectal $(17=14.91 \%)$. Diagnosis was made at the National Cancer Institute of Colombia, to whom applied the FACT-G and EQ-5D scale. Descriptive analyses were used to summarize the variables, methods of multivariate analysis, factor analysis and multidimensional scaling were used to assess patterns of correlation between the variables of each of the two scales used. Results: The dimensions that showed acceptable correlation values were physical well-being and emotional well-being of the FACT- $G$ with anxiety and depression of the EQ- 5D scale. Factor analysis yielded two factors: (a) anxiety / depression or the EQ-5D is better grouped with the components of the FACT- G, and (b) physical symptoms of FACT-G with the components of the EQ-5D. Multidimensional scaling analysis suggested that each scale measured different dimensions of the construct quality of life. Conclusion: The FACT-G is oriented to emotional and social interaction, while the EQ-5D is oriented to aspects of physical and functionality. Keywords: quality life; EQ-5D; FACT-G; cancer.

\section{Resumo}

Objetivo: avaliar patrões de correlações entre as diferentes dimensões de uma escala que mede a qualidade de vida (FACT-G) e outra que mede a saúde auto-percebida e a qualidade de vida (EQ-5D) em pacientes com cáncer. Material e métodos: estudo descritivo que avaliou a 114 pacientes com câncer de mama $(40=35.09 \%)$, gástrico $(26=22.8 \%)$, próstata $(22=19.3 \%)$, cólon/reto $(17=14.91 \%)$ aos quais se lhes aplicaram as escalas FACT-G e EQ-5D. Utilizaram-se técnicas de estatística descritiva para resumir as variáveis, métodos de análise multivariado, análise fatorial e escalamento multidimensional. Resultados: as dimensões que mostraram valores de correlação aceitável foram bem-estar físico e emocional da FACT-G com angústia e depressão da escala EQ-5D. A análise fatorial revelou dois fatores: (a) angústia/depressão da EQ-5D agrupa-se melhor com os componentes da FACT-G, (b) sintomas físicos com os componentes da EQ-5D. O escalamento multidimensional sugeriu que cada escala mediu dimensões diferentes do constructo qualidade de vida. Conclusão: a avaliação de qualidade de vida da FACT-G parece orientar-se a aspetos emocionais e de interação social, enquanto que a EQ-5D orienta-se a aspetos de bem-estar físico e funcionalidade.

Palavras-chave: qualidade de vida, EQ-5D, FACT-G, câncer.

Según el Plan Nacional para el Control del Cáncer en Colombia 2010-2019, el cáncer constituye un grupo de enfermedades con grandes repercusiones sociales, económicas y emocionales. La carga creciente del cáncer en el perfil de salud de los colombianos amerita intervenciones oportunas, certeras y coordinadas para lograr el impacto esperado en los ámbitos poblacional e individual sobre su incidencia, discapacidad, calidad de vida y mortalidad (Ministerio de Protección Social-Instituto Nacional de Cancerología, 2010a).

Dentro de los estudios sobre desenlaces en cáncer (Potter et al., 2010; Tegels, De Maat, Hulsewé, Hoofwijk, \& Stoot, 2014; Torres-Mesa, Oliveros, Mesa, Olaya, \& Sánchez, 2014), ha llamado la atención la evaluación de la calidad de vida, que está definida por la Organización Mundial de la Salud como la percepción del individuo sobre su posición en la vida dentro del contexto cultural y el sistema de valores en el que vive y con respecto a sus metas, expectativas, normas y preocupaciones. Es un concepto extenso y complejo que engloba la salud física, el estado psicológico, el nivel de independencia, las relaciones sociales, las creencias personales y la relación con las características sobresalientes del entorno (WHOQOL Group, 1997).

Aunque existen otras definiciones de calidad de vida, algunos autores han criticado este concepto. Algunos de ellos son Moons, Budts \& De Geest (2006), cuya revisión analiza algunas conceptualizaciones relacionadas con la calidad de 
vida, como la vida normal, la utilidad social, la utilidad, la felicidad/afecto, la satisfacción con la vida, la satisfacción con dominios específicos, los logros de objetivos personales y las capacidades naturales, las cuales se han referido dentro de la literatura biomédica y de enfermería. Moons et al. señalan los problemas involucrados en este tipo de conceptualizaciones, por ejemplo, confundir el estado de salud con la calidad de vida y con el funcionamiento, reconocer si la calidad de vida posee más elementos objetivos o subjetivos, el efecto que produce el paso del tiempo, etc. A partir de esta revisión, los autores concluyeron que la satisfacción con la vida, como una evaluación subjetiva de la vida personal, se relaciona más con el concepto de calidad de vida.

Al cuestionarse sobre la naturaleza multidimensional del concepto de calidad de vida, Sánchez, Sierra e Ibáñez (2012) analizaron las respuestas de pacientes con cáncer a la pregunta qué es calidad de vida. En el análisis lexicográfico se encontró que la calidad de vida tendría ocho dimensiones subyacentes (espiritualidad, familia, sistema de salud, social, funcional, síntomas, componente económico y componente emocional).

A partir de la caracterización de las dimensiones de la calidad de vida, se han desarrollado instrumentos que pretenden medirlas, como la EORTC QLQ-C30 (Aaronson et al., 1993) y la SF-36 (Ware \& Sherbourne, 1992). Para el presente estudio, el interés se centró en la escala EQ-5D, del EuroQoL Group (2013), y la escala FACT-G, de la Functional Assessment for Chronic Illness Therapy (FACIT) (Cella \& Nowinski, 2002).

La EQ-5D es un instrumento genérico que mide estados de salud, a partir de un sistema descriptivo que comprende cinco dimensiones: movilidad, autocuidado, actividades cotidianas, dolor/ malestar y ansiedad/depresión. Cada una de estas dimensiones tienen tres respuestas: sin problema (1), algunos problemas (2) y problemas extremos (3). El paciente señala cuál es más apropiada para cada dimensión que represente su estado de salud.
El instrumento también tiene una escala análoga visual (EQ VAS, por su sigla en inglés) en la que el paciente señala en una escala de 0 ("peor estado de salud imaginable") a 100 ("mejor estado de salud imaginable") como percibe su estado de salud actual (EuroQoL Group, 2013). Este instrumento se ha aplicado en varios grupos de poblaciones y generalmente se utiliza para estimar años de vida ajustados por calidad (QUALY, por su sigla en inglés) y en la toma de decisiones para el uso de nuevas tecnologías sanitarias (Cañón \& Rodríguez, 2011; Herdman, Badia \& Berra, 2001).

La FACT-G es una escala desarrollada por la FACIT y fue validada en Colombia con un total de 473 pacientes con distintos tipos de cáncer, que asistieron al Instituto Nacional de Cancerología (Sánchez, Ballesteros \& Arnold, 2011). Esta escala evalúa el concepto de calidad de vida en cuatro dimensiones: estado físico general de salud, ambiente familiar y social, estado emocional y capacidad de funcionamiento personal. Los ítems se califican en una escala tipo Likert $u$ ordinal entre 0 y $4(0=$ nada, $1=$ un poco, $2=$ algo, $3=$ mucho, 4 = muchísimo). La calificación se realiza con un algoritmo propuesto por la FACIT, con mayores puntajes que indican una mejor calidad de vida y fue diseñada para ser autoaplicable o por medio de un entrevistador, y también por vía telefónica (Cella \& Nowinski, 2002).

Aunque las dos escalas buscan medir la calidad de vida, es necesario observar que la EQ-5D es una escala genérica; mientras que la FACT-G es una escala específica para cáncer. Además, desde su diseño, hay diferencias en el número y el tipo de dimensiones que las integran, así como en la complejidad de las preguntas y la forma de su calificación, lo cual puede sugerir que las dos escalas tienen dos concepciones distintas sobre qué es la calidad de vida. De acuerdo con esto, es necesario conocer desde cuál perspectiva fueron construidas estas escalas y cuáles dimensiones son compartidas entre ellas, para así utilizar la más adecuada para los objetivos de las investigaciones que se quieran 
plantear más adelante. El presente estudio tiene como objetivo evaluar los patrones de correlaciones entre las diferentes dimensiones de las escalas FACT-G y EQ-5D, en una muestra de pacientes con distintos tipos de cáncer.

\section{Metodología}

\section{Participantes}

Se evaluaron 114 pacientes con diagnóstico confirmado histopatológicamente de cáncer de mama, próstata, cuello uterino, estómago y colon-recto, candidatos a cirugía con intención curativa, mayores de 18 años de edad y con nacionalidad colombiana. Se excluyeron pacientes que tuvieran alteraciones sensoriales o cognitivas que les impidieran responder adecuadamente las escalas y quienes ya habían sido intervenidos quirúrgicamente con otras patologías oncológicas. El contacto de los pacientes se realizó en la consulta externa del Instituto Nacional de Cancerología ESE o por vía telefónica, por medio de un entrevistador. Tras el asentimiento verbal, se aplicaron las escalas FACT general (FACT-G) y la escala genérica de calidad de vida EuroQoL 5D (EQ-5D).

\section{Análisis estadístico}

Se utilizaron técnicas de estadística descriptiva para resumir las variables del estudio: medias y medianas junto con sus medidas de dispersión para variables continuas y porcentajes para variables categóricas. La selección de medias o medianas obedeció a la presencia de simetría en la distribución de la variable.

Para evaluar los patrones de correlación entre las variables de cada una de las dos escalas utilizadas en el estudio (las cinco dimensiones de la escala EQ-5D y la subescala EQ VAS y las cuatro de la escala FACT-G: bienestar físico, bienestar sociofamilar, bienestar emocional y bienestar funcional) se utilizaron métodos de análisis multivariado. Dada la naturaleza ordinal de ambas escalas, se usaron matrices de correlación policóricas para estimar los coeficientes de correlación más apropiados a la naturaleza de las variables estudiadas.

Sobre la base de las matrices de correlación policórica, se efectuó un análisis factorial para evaluar los eventuales patrones de asociación entre los seis componentes de la escala EQ-5D y los cuatro de la escala FACT-G. Para los análisis factoriales se utilizaron métodos de residuos mínimos. Para seleccionar la mejor representación dentro de cada eje, se efectuaron métodos de rotación ortogonal y oblicua (varimax y promax, respectivamente).

Adicionalmente, se practicó un escalamiento multidimensional con un método de "mayorización iterativa" que se puede efectuar con el procedimiento Smacof del lenguaje R. Los análisis incluidos en el presente estudio hacen parte de un proyecto de investigación Calidad de vida relacionada con el tratamiento quirúrgico en pacientes con cáncer de mama, cuello uterino, próstata, estómago, colon y recto, aprobado por el Comité de Ética del Instituto Nacional de Cancerología ESE, en el 2012.

\section{Resultados}

La muestra de pacientes evaluada en este estudio fue de 114 y estuvo constituida por el $56.1 \%$ de mujeres $(N=64)$. La mayoría de los pacientes presentaron cáncer de mama $(35.09 \% ; N=40)$ y cáncer gástrico $(22.81 \% ; N=26)$ (tabla 1$)$. La edad tuvo una media de 58.2 años $(D E=12.9)$. De acuerdo con el estrato socioeconómico, la distribución fue la siguiente: estrato $1: 15.79 \%(N=18)$; estrato 2: $42.11 \%(N=48)$; estrato $3: 35.96 \%$ $(N=41)$, y estrato $4: 6.14 \%(N=7)$. Los pacientes tuvieron una mediana de escolaridad de 6 años cursados (rango entre 0 y 22). La mayoría de los pacientes eran casados $(42.11 \% ; N=48)$. Los demás estados civiles, por orden de frecuencia, tuvieron la siguiente distribución: unión libre: $26.32 \%$ $(N=30)$; soltero: $16.67 \%(N=19)$; viudo: $9.65 \%$ $(N=11)$, y separado $5.26 \%(N=6)$. 
Tabla 1

Distribución de los pacientes según localización del cáncer

\begin{tabular}{lrr}
\hline \multicolumn{1}{c}{ Tipo de cáncer } & $N$ & \multicolumn{1}{c}{$\%$} \\
\hline Mama & 40 & 35.09 \\
Gástrico & 26 & 22.81 \\
Próstata & 22 & 19.30 \\
Colon-recto & 17 & 14.91 \\
Cuello uterino & 9 & 7.89 \\
Total & 114 & 100.00
\end{tabular}

El análisis multivariado realizado con las 10 dimensiones estudiadas (figura 1) mostró valores de correlación modestos (la tabla 2 señala los valores de correlación de la matriz policórica). La dimensión de bienestar físico de la escala FACT-G muestra valores de correlación aceptables con las dimensiones de dolor/malestar (-.564), actividades cotidianas (-.489) y angustia/depresión $(-.435)$ de la EQ-5D. También se observó una correlación aceptable entre la dimensión de bienestar emocional de la FACT-G con la de angustia/depresión $(-.529)$ de la EQ-5D.

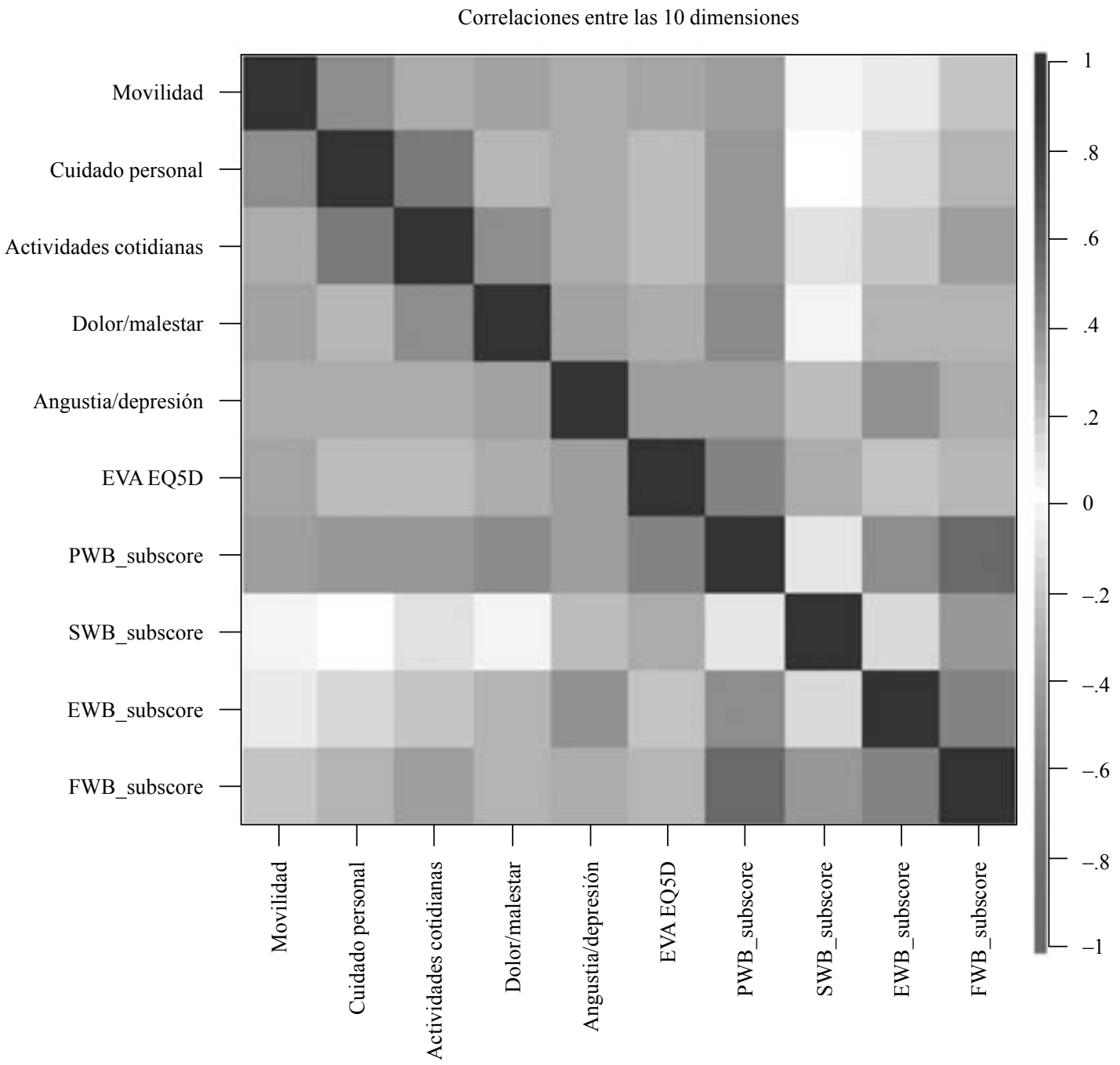

Figura 1. Mapa de correlaciones ente las dimensiones de las escalas FACIT-G y EQ-5D 
Tabla 2

Valores de correlación entre las dimensiones de las escalas FACT-G y EQ-5D

\begin{tabular}{lcccccccccc}
\hline & $\begin{array}{c}\text { Movili- } \\
\text { dad }\end{array}$ & $\begin{array}{c}\text { Cuidado } \\
\text { perso- } \\
\text { nal }\end{array}$ & $\begin{array}{c}\text { Activida- } \\
\text { des coti- } \\
\text { dianas }\end{array}$ & $\begin{array}{c}\text { Dolor/ } \\
\text { males- } \\
\text { tar }\end{array}$ & $\begin{array}{c}\text { Angus- } \\
\text { tia/de- } \\
\text { presión }\end{array}$ & $\begin{array}{c}\text { Global } \\
\text { EQ5D }\end{array}$ & $\begin{array}{c}\text { FACT-G } \\
\text { físicos }\end{array}$ & $\begin{array}{c}\text { FACT-G } \\
\text { sociofa- } \\
\text { miliar }\end{array}$ & $\begin{array}{c}\text { FACT-G } \\
\text { nal }\end{array}$ & $\begin{array}{c}\text { FACT-G } \\
\text { funcio- } \\
\text { nal }\end{array}$ \\
\hline Movilidad & 1.000 & .395 & .294 & .325 & .262 & -.400 & -.429 & -.023 & -.084 & -.231 \\
Cuidado personal & .395 & 1.000 & .472 & .237 & .269 & -.291 & -.460 & .006 & -.176 & -.327 \\
Actividades & & & & & & & & & & \\
Cotidianas & .294 & .472 & 1.000 & .385 & .290 & -.274 & -.489 & -.112 & -.224 & -.438 \\
Dolor/malestar & .325 & .237 & .385 & 1.000 & .319 & -.349 & -.564 & -.053 & -.298 & -.324 \\
Angustia/depresión & .262 & .269 & .290 & .319 & 1.000 & -.435 & -.435 & -.277 & -.529 & -.351 \\
Global EQ5D & -.400 & -.291 & -.274 & -.349 & -.435 & 1.000 & .444 & .260 & .200 & .247 \\
FACT-G físicos & -.429 & -.460 & -.489 & -.564 & -.435 & .444 & 1.000 & .070 & .407 & .551 \\
FACT-G sociofamiliar & -.023 & .006 & -.112 & -.053 & -.277 & .260 & .070 & 1.000 & .122 & .334 \\
FACT-G emocional & -.084 & -.176 & -.224 & -.298 & -.529 & .200 & .407 & .122 & 1.000 & .422 \\
FACT-G funcional & -.231 & -.327 & -.438 & -.324 & -.351 & .247 & .551 & .334 & .422 & 1.000 \\
\hline
\end{tabular}

Para establecer el número de ejes que mejor resumiera el conjunto de 10 variables dentro del análisis factorial se utilizó un análisis paralelo. De acuerdo con este método, se incluyeron dos ejes o factores. Los resultados del análisis factorial se presentan en la tabla 3. Así, el componente relacionado con angustia/depresión de la escala EQ-5D se agrupó mejor con la mayoría de componentes de la escala FACT-G (bienestar social, bienestar emocional y bienestar funcional); mientras que el componente de bienestar físico de la escala FACT-G se agrupó en la dimensión mejor representada por la escala EQ-5D con las dimensiones de cuidado personal, movilidad, actividades cotidianas y dolor/ malestar de la EQ-5D. El ítem que midió estado de salud tuvo una representación ligeramente mejor en el factor que agrupó la mayoría de componentes de la escala EQ-5D.

En relación con el escalamiento multidimensional, se encontró un valor de estrés no métrico de $2,75 \times 10^{-6}$, lo que indica un ajuste adecuado del análisis sobre una estructura bidimensional. De acuerdo con los porcentajes de estrés por punto, los componentes de las escalas que mostraron menor
Tabla 3

Estructura factorial en dos dimensiones (rotación promax)

\begin{tabular}{|c|c|c|c|c|}
\hline & $\begin{array}{c}\text { Factor } \\
1^{*}\end{array}$ & $\begin{array}{c}\text { Factor } \\
2^{*}\end{array}$ & $\begin{array}{l}\text { Comu- } \\
\text { nalidad }\end{array}$ & $\begin{array}{c}\text { Unici- } \\
\text { dad }\end{array}$ \\
\hline FACT-G físicos & -.75 & .37 & .7 & .3 \\
\hline Cuidado personal & .73 & & .54 & .46 \\
\hline Movilidad & .71 & & .51 & .49 \\
\hline $\begin{array}{l}\text { Actividades } \\
\text { cotidianas }\end{array}$ & .66 & & .48 & .52 \\
\hline Dolor/malestar & .61 & & .44 & .56 \\
\hline EVAEQ5D & -.49 & .39 & .39 & .61 \\
\hline $\begin{array}{l}\text { FACT-G } \\
\text { sociofamiliar }\end{array}$ & & .71 & .53 & .47 \\
\hline Angustia/depresión & .32 & -.69 & .58 & .42 \\
\hline FACT-G emocional & & .68 & .5 & .5 \\
\hline $\begin{array}{l}\text { FACT-G } \\
\text { funcionalidad }\end{array}$ & -.4 & .61 & .54 & .46 \\
\hline
\end{tabular}

* Solo se presentan cargas factoriales > .3.

calidad de ajuste fueron los relacionados con la escala FACT-G (los valores de estrés estuvieron entre 11.6 y $28.3 \%$ ). Los porcentajes de estrés para 
los componentes de la escala EQ-5D estuvieron entre 1.600 y $1.603 \%$.

La figura 2, correspondiente al escalamiento multidimensional, sugiere que cada escala mide dimensiones diferentes; también se encontró que el ítem que mide estado de salud (es uno en el que el paciente cuantifica su estado de salud en la escala EQ-5D) está alejado de las dos agrupaciones anteriores. Ello sugiere que mide aspectos que los dos conglomerados que representan las dos escalas utilizadas no incorporan.

\section{Discusión}

El objetivo de este estudio fue evaluar los patrones de correlaciones entre las dimensiones de la FACT-G y de la EQ-5D en una muestra de pacientes con diferentes tipos de cáncer. Los resultados mostraron que, a pesar de que las dimensiones se agruparon en dos factores, el escalamiento multidimensional señaló que cada una de las escalas evalúa aspectos distintos de la calidad de vida. En el análisis factorial se sugiere que hay dos componentes que sobresalen dentro de la evaluación de calidad de vida: el primero es el físico y de funcionalidad, que se representó mejor en las dimensiones de la escala EQ-5D, junto con la dimensión de bienestar físico de la FACT-G, y el segundo es el emocional y de interacción social, mejor representado por las dimensiones de la FACT-G, junto con la dimensión de angustia/depresión de la EQ-5D. De acuerdo con esto, se podría decir que las dos escalas tienen en común la evaluación de aspectos funcionales y emocionales.

Sin embargo, al observar los resultados del escalamiento multidimensional, se sugiere que las escalas recogen aspectos diferentes del constructo calidad de vida. La agrupación entre sí de las dimensiones de la escala EQ-5D se enfoca más en aspectos relacionados con la funcionalidad y el poder hacer actividades cotidianas, mientras que las dimensiones de la FACT-G se agrupan en torno a aspectos emocionales y de interacción social. Estas agrupaciones se encuentran separadas entre sí y, además, el ítem correspondiente a estado de salud (EQ VAS) presenta un mayor distanciamiento de estas escalas, es decir, una mayor disimilaridad.

Existen pocos estudios de comparación de las escalas EQ-5D y FACT-G en diversas poblaciones. En una validación de la EQ-5D al idioma chino

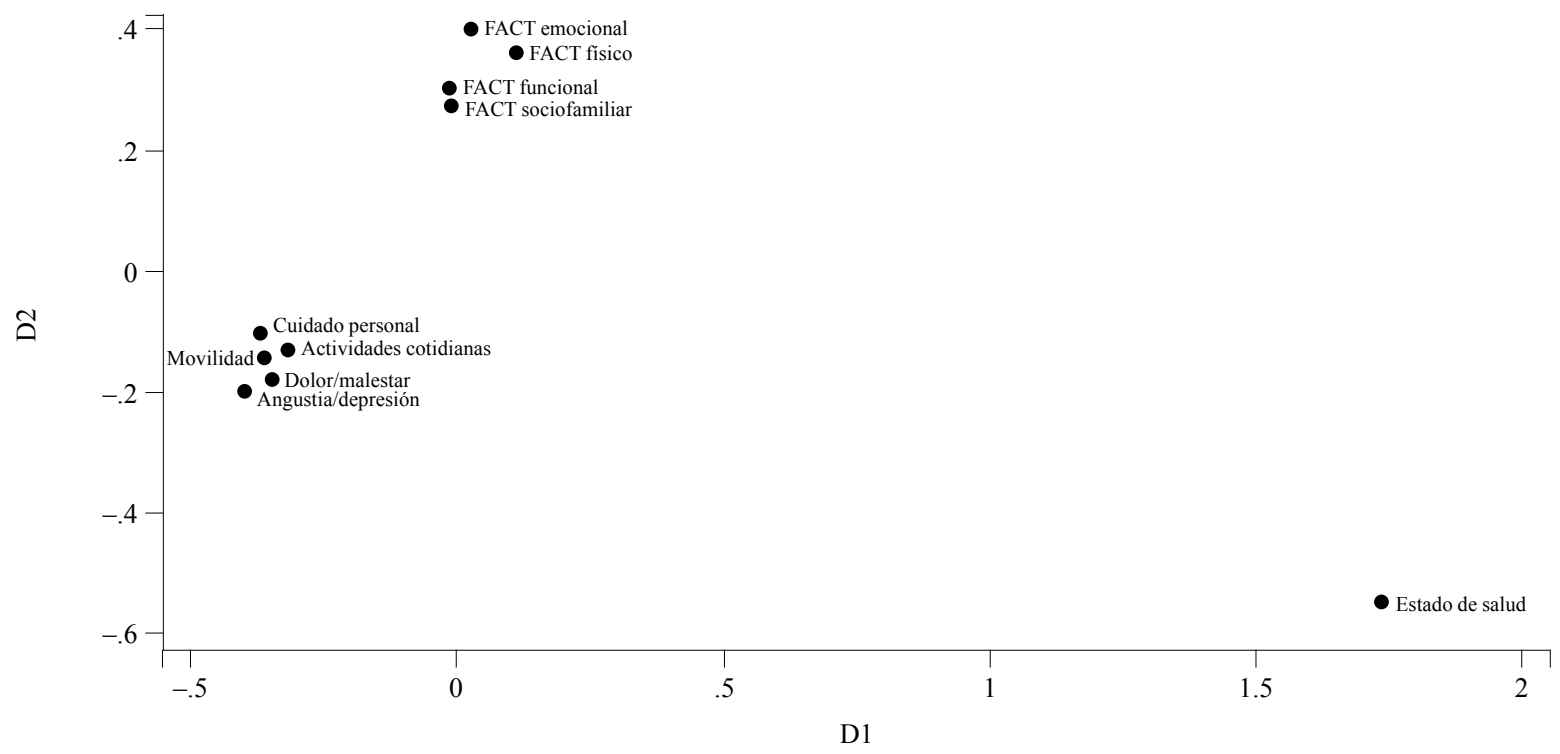

Figura 2. Escalamiento multidimensional en dos dimensiones 
e inglés en Singapur (Gao et al., 2009) se evaluó la validez de convergencia y divergencia entre la EQ-5D y la FACT-G, y se encontraron fuertes correlaciones entre los ítems de ansiedad/depresión de la EQ-5D con la subescala de bienestar emocional de la FACT-G ( $p=-.52$ y -.65 para la versión en inglés y en chino, respectivamente). En otro estudio (Teckle, 2013), a partir de modelos de regresión, se utilizaron las respuestas de la FACT-G para estimar los índices de utilidades de la EQ-5D y la SF-6D, teniendo en cuenta que la primera es una escala específica para el cáncer y las otras son escalas genéricas basadas en la preferencia. El objetivo del estudio de Teckle (2013) era facilitar las evaluaciones económicas de los tratamientos oncológicos.

Una razón por la cual estas escalas parecen evaluar aspectos distintos de la calidad de vida tiene que ver con la forma como estas se han caracterizado, siendo la EQ-5D genérica y la FACT-G específica de la enfermedad. Ferrans (2010) señala que los instrumentos genéricos permiten comparar entre la población general y entre grupos de enfermedad; no obstante, estas medidas son demasiado amplias y abordan aspectos de la vida superficialmente, en contraste con las específicas de la enfermedad, que son capaces de detectar cambios específicos de los síntomas (Ferrans, 2010). Esto se podría observar en la manera cómo se califican los ítems en cada una de ellas. La FACT-G es más amplia en las preguntas y en las opciones de respuesta para cada uno de los dominios; mientras que la EQ-5D se limita a tres opciones de respuesta por cada dominio.

Cuando se revisó la literatura sobre estas dos escalas, llamó la atención que los estudios con la EQ-5D se orientaban hacia cómo esta puede utilizarse en la estimación de costo-utilidad y de QUALY, una de las variables que ha ganado importancia en la toma de decisiones para evaluación e implementación de nuevas tecnologías sanitarias (Cañon \& Rodríguez, 2011; Kontodimopoulos, Aletras, Paliouras, \& Niakas, 2009; Pickard, 2007).
En conclusión, aunque las escalas EQ-5D y FACT-G pretenden medir el mismo constructo, muestran dos abordajes distintos de la calidad de vida: una como funcionalidad y la otra como bienestar emocional. La utilización de una u otra dependerá del objetivo del estudio. No obstante, es necesario continuar con los estudios de comparación entre los instrumentos que miden calidad de vida y las dimensiones que describen mejor el constructo. Esto no solo debe quedarse en el nivel de los análisis estadísticos y teorización, sino que debe tener aplicabilidad en la práctica clínica.

\section{Referencias}

Aaronson, N. K., Ahmedzai, S., Bergman, B., Bullinger, M., Cull, A., Duez, N. J., ... de Haes, J. C. (1993). The European Organization for Research and Treatment of Cancer QLQ-C30: A quality-of-life instrument for use in international clinical trials in oncology. Journal of the National Cancer Institute, 366-374.

Cañón, O. \& Rodríguez, C. (2011). Introducción al uso de QALYs y EQ-5D en la evaluación de tecnologías en Colombia. Revista CIFE, 13(19), 33-49.

Cella, D., \& Nowinski, C. J. (2002). Measuring quality of life in chronic illness: The functional assessment of chronic illness therapy measurement system. Archives of Physical Medicine and Rehabilitation, 83(12 suppl 2), s10-7.

EuroQoL Group. (2013). User guide: Basic information on how to use the EQ-5D-3L instrument version 5.0. s. 1.: autor.

Ferrans, C. (2010). Advances in measuring quality og life outcomes in cancer care. Seminars in Oncology Nursing, 26(1), 2-11.

Gao, F., Ng, G., Cheung, Y., Thumboo, J., Pang, G., Koo, W., ... Goh, C. (2009). The Singaporean English and Chinese versions of the EQ-5D achieved measurement equivalence in cancer petients. Journal of Clinical Epidemiology, 62, 206-213. 
Herdman, M., Badia, X., \& Berra, S. (2001). El EuroQoL-5D: una alternativa sencilla para la medición de la calidad de vida relacionada con la salud en atención primaria. Atención Primaria, 28(6), 425-429.

Koller, M., \& Lorenz, W. (2002). Quality of life: a deconstruction for clinicians. Journal of the Royal Society of Medicine, 481-488.

Kontodimopoulos, N., Aletras, V. H., Paliouras, D., $\&$ Niakas, D. (2009). Mapping the cancer-specific EORTC QLQ-C30 to the preference-based EQ-5D, SF-6D, and 15D instruments. Value in Health, 12(8), 1151-1157.

Ministerio de Protección Social-Instituto Nacional de Cancerología ESE. (2010a). Plan Nacional para el Control del Cáncer. Bogotá: autores.

Ministerio de Protección Social-Instituto Nacional de Cancerología. (2010b). Plan Nacional para el Control del Cáncer en Colombia 2010-2019. Bogotá: autores.

Moons, P., Budts, W., \& De Geest, S. (2006). Critique on the conceptualisation of quality of life: A review and evaluation of different conceptual approaches. International Journal of Nursing Studies, 891-901.

Pickard, A. W. (2007). Impact of cancer on Health related Quality of life: Evidence using the EQ5D. Badia.

Potter, S., Brigic, A., Whiting, P., Cawthorn, S., Avery, K., Donovan, J., \& Blazeby, J. (2010). Reporting clinical outcomes of breast reconstruction: A. Journal of National Cancer Institute, 31-46.

Sánchez, R., Ballesteros, M., \& Arnold, B. (2011). Validation of the FACT-G scale for evaluating quality of life in cancer patients in Colombia. Quality of Life Research, 20, 19-29.

Sánchez, R., Sierra, F., \& Ibáñez, C. (2012). Dimensiones que conforman el constructo de calidad de vida en pacientes con cáncer. Revista Colombiana de Cancerología, 100-109.

Teckle, P. M.-C. (2013). Mapping the FACT-G cancer-specific quality of life instrument to the EQ-5D and SF-6D. Health and Quality of life Outcomes, 11, 203.

Tegels, J., De Maat, M., Hulsewé, K., Hoofwijk, A., \& Stoot, J. (2014). Improving the outcomes in gastric cancer surgery. World Journal of Gastroenterology, 13692-13704.

Torres-Mesa, P. A., Oliveros, R., Mesa, J., Olaya, N., \& Sánchez, R. (2014). Desenlaces del manejo no quirúrgico posterior a neoadyuvancia del cáncer localmente avanzado de recto. Revista Colombiana de Cancerología, 109-119.

Ware, J. E. \& Sherbourne, C. D. (1992). The MOS 36-item short-form health survey (SF-36). I. Conceptual framework and item selection. $\mathrm{Me}$ dical Care, 30(6), 473-483.

WHOQOL Group. (1997). Measuring quality of life. Geneva: World Health Organization.
Fecha de recepción: 10 de julio de 2014 fecha de aceptación: 15 de abril de 2015 